\title{
Natural Course of Subsequent Pregnancy after Peripartum Cardiomyopathy
}

\author{
Francisco Manes Albanesi F, Tatiana Tavares da Silva
}

Rio de Janeiro, RJ - Brazil

\begin{abstract}
Objective - To assess the effect of subsequent pregnancy after peripartum cardiomyopathy (PPCM) on maternal and fetal outcome.

Methods - Prospective study of 34 patients with the diagnosis of PPCM (mean age $=26 y e a r s)$. At the time of first diagnosis 5 were in NYHA functional class (FC) II for heart failure, one in FC III and 28 in FC IV. After clinical treatment, patients were advised to avoid new pregnancies and a follow-up was obtained.
\end{abstract}

Results - There were 12 (35.3\%) subsequent pregnancies in patients (pt) aged 19 to 44 years (mean 32), divided into two groups: GI: 6 pts who had normalized their heart size and GII: 6 pts with persistent cardiomegaly. GI had initially mild clinical manifestations ( 3 were in FC II, 1 in FCII and 2 in FC IV) and complete recovery of cardiac function (FCI). A new pregnancy was well-tolerated in 5 (83.3\%); 1 pt presented with preeclampsia, and progressed to FC II. Presently, 5 ptare in FCI and 1 in FC II. GII pts had more severe heartfailure at the onset of PPCM (1 pt in FC II and 5 in FCIV); during follow-up, 4 pt were in FCI and 2 in FC II. A new pregnancy was well tolerated in all of them, but the eldest, who had had 2 pregnancies and had a progressive worsening of clinical status, dying 8 years after the last pregnancy and 13 years after the diagnosis of PPCM. The remaining 5 pt are still alive, 3 in FC I and 2 in FC II, with worsening of FC in 1. Subsequent pregnancies occurred 37 years after clinical treatment of PPCM and no fetal distress was observed.

Conclusion - Subsequent pregnancies are well-tolerated after PPCM, but not devoid of risk. No fetal distress was observed. A minimum interval of 3 years after the recovery of function seems to be safe for subsequent pregnancies.

Keywords: peripartum cardiomyopathy, subsequent pregnancy

Universidade do Estado do Rio de Janeiro - UERJ.

Mailing adress: Francisco Manes Albanesi $F^{\circ}$ - Av. 28 de Setembro, 77 - $2^{\circ}$ - 20551-030

- Rio de Janeiro, RJ - Brazil
Peripartum cardiomyopathy (PPCM) is a disease of unknown etiology, characterized by systolic impairment, which occurs in previously healthy women (no preexisting cardiovascular diseases) mainly during the last three months of pregnancy and the first 6 months after delivery ${ }^{1-7}$. Incidence of the disease is variable; in the USA it is reported to be about $1 / 15,150$ of all pregnancies ${ }^{8}$, which means less than $1 \%$ of cardiovascular diseases associated to pregnancy. The risk of subsequent pregnancies after improvement in clinical status is still debatable ${ }^{9-10}$. In patients with partial functional recovery, Oakley ${ }^{9}$ discourages subsequent pregnancies, and for those who have complet normalization of left ventricular function, he recommends an interval of 3 to 5 years. The persistence of left ventricular dysfunction, even mild, contraindicates subsequent pregnancies ${ }^{5,9,10}$. In patients who do not recover function, subsequent pregnancies are associated to PPCM exacerbation, with a 50\% maternal mortality risk ${ }^{1,2,10}$. Subsequent pregnancies are accepted for patients with functional recovery (11), but a high risk of disease relapse and maternal morbidity is expected ${ }^{10}$. The maternal risks are higher for patients with left ventricular dysfunction and persistent cardiomegaly; however, risks are also increased for those with normal function ${ }^{13}$. Accordingly, subsequent pregnancies are to be discouraged in patients with PPCM and left ventricular dysfunction. Patients who recover function after PPCM should be advised that a subsequent pregnancy is not devoid of risk, and conception should thus be discussed among the family and physicians ${ }^{10}$. Since the matter is still controversial, we decided to study the clinical course of a subsequent pregnancy in patients with PPCM in whom pregnancy had been discouraged until complete recovery of clinical status.

\section{Methods}

PPCM was defined as a heart failure syndrome of unknown cause occurring in the last three months of pregnancy and first six months post partum, in the absence of any preexisting cardiovascular disease ${ }^{1-4}$. 
From 1/1976 to 12/1996, we followed 34 women with PPCM, most non-white $(9 / 12-68.1 \%)$, with age ranging from 16 to 41 (mean 26) years. First diagnosis was made during pregnancy (from the $32^{\text {nd }}$ week until delivery) in $4(11.76 \%)$ patients and in $30(88.24 \%)$ patients, diagnosis was made after delivery ( 11 in the first month in the puerperium while in the remaining pts from the 2 nd to the $6^{\text {th }}$ month). At the time of diagnosis, NYHA functional class (FC) for heart failure (fig. 1) was the following: FC II: 5 patients, FC III, one patient, FCIV, 28 patients. Treatment consisted of diuretics, digitalis and angiotensin-converting enzyme (ACE) inhibitor and anticoagulants, the last two only used after delivery. After treatment, patients improved into FC I (22 patients), FC II (9 patients) and FC III (3 patients). They were advised not to get pregnant, especially patients with persistently increased heart size and abnormal left ventricular function. In this particular group of patients, after discussion with the family, tubal ligation was offered as a means of contraception, since it has a high index of success and no effects on cardiovascular system, such as those produced by oral contraceptives. Patients who recovered function were advised to postpone pregnancy for at least two to three years. Twelve patients who did not follow that advice and had a subsequent pregnancy during this period consisted of our study group. The study was therefore prospective, with the patients' follow-up occurring every 3 weeks during pregnancy and postpartum, after which they were seen every 6 months.

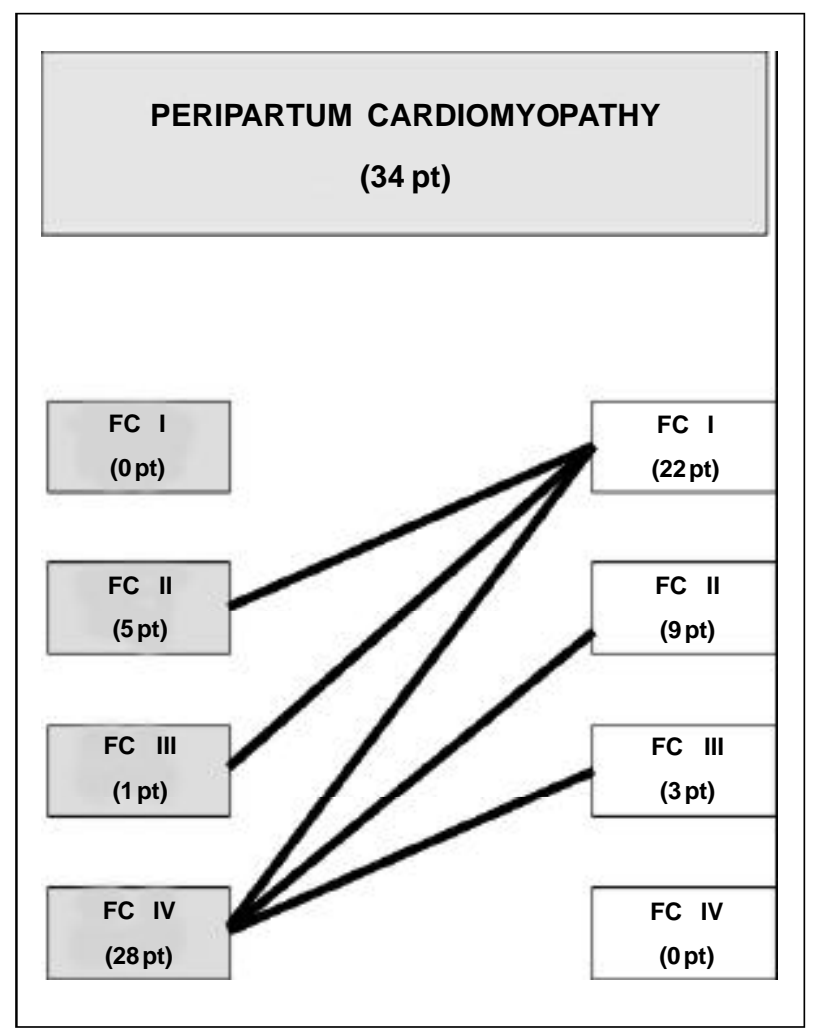

Fig. 1 - Functional class at the onset of PPCM and after improvement in clinical condition in 34 patients.
Left ventricular dysfunction was defined as the presence of signs and symptoms of heart failure and increased heart size observed in one or more exams (electrocardiography, chest X-ray, echocardiography). Left ventricular diastolic and systolic diameters, ventricular septum and posterior wall thickness, left atrial size and the degree of left ventricular dysfunction were defined by echocardiography.

Patients were followed after the diagnosis of PPCM and when a subsequent pregnancy occurred, left ventricular function was once again assessed by echocardiography. Patients were then divided into two groups, according to the presence of dysfunction: Group I (GI) consisted of patients with normal function who were clinically in FC I; Group II consisted of patients with increased heart chambers and left ventricular dysfunction (4 patients were in FC I and 2 were in FC II) (fig. 2).

\section{Results}

Twelve patients had subsequent pregnancies and, among these, there were 16 pregnancies. Their age ranged from 19 to $44($ mean $=32)$ years old and nine of them were non-white. Each group consisted of six patients (table I and fig. 2). NYHAFC at the time of first diagnosis of PPCM for GI patients (normal left ventricular function) was: FC II, 3 patients, FC III, 1 patient and FC IV, 2 patients. There were 9 pregnancies in this group (one patient had 2 subsequent pregnancies, another had 3 more), all of them well tolerated, except for one, who developed preeclampsia associated to MMCP relapse. This patient deteriorated from FC I into II. Preeclampsia in this patients was defined as mild (blood pressure $<160 / 100 \mathrm{mmHg}$, proteinuria $<1 \mathrm{~g} / 24 \mathrm{~h}$, minimal pretibial edema). Presently, all patients are alive, 5 in FC I and 1 in FC II.

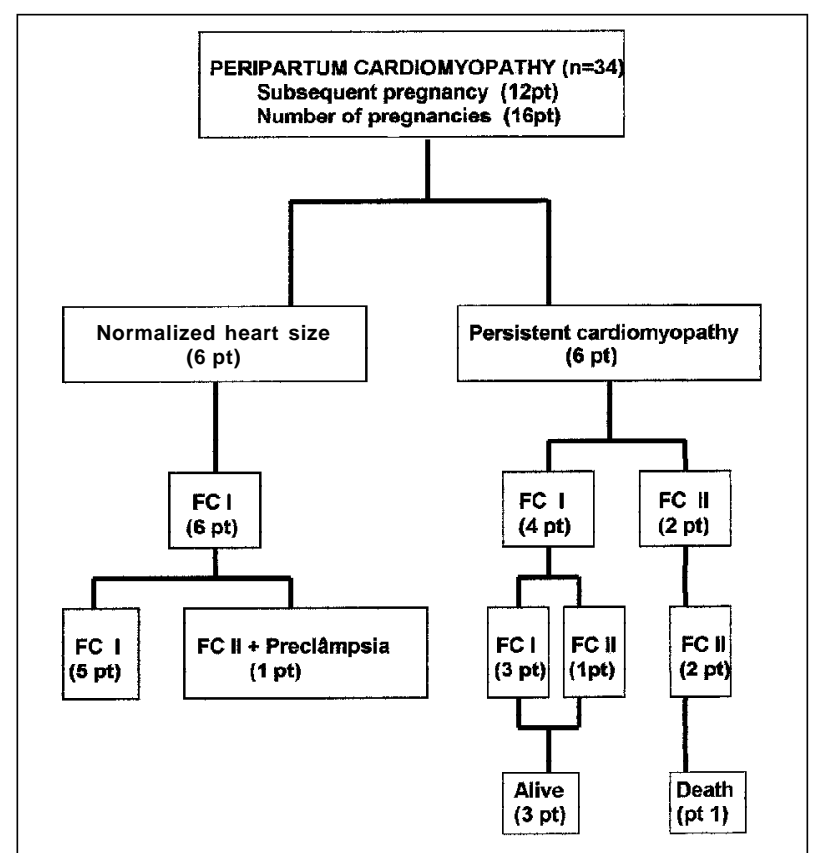

Fig. 2 - Clinical course of the 12 patients with PPCM and 16 subsequent pregnancies, divided into two groups: group I with normalized heart size, and group II with persistent cardiomegaly. 


\begin{tabular}{|c|c|c|c|c|c|}
\hline $\begin{array}{l}\text { Age (years) at } \\
\text { the onset of PPCM }\end{array}$ & $\begin{array}{l}\text { Pregnancy number at the } \\
\text { onset of PPCM initial FC }\end{array}$ & $\begin{array}{l}\text { Initial of } \\
\text { FC }\end{array}$ & $\begin{array}{l}\text { FC at subsequent } \\
\text { pregnancy }\end{array}$ & $\begin{array}{c}\text { Number of subsequent } \\
\text { pregnancies }\end{array}$ & $\begin{array}{c}\text { Clinical course in } \\
\text { subsequent pregnancies }\end{array}$ \\
\hline 18 & I & IV & I & 1 & IHS \\
\hline 41 & II & IV & II & 1 & IHS \\
\hline 40 & VI & IV & IV & 1 & IHS \\
\hline 32 & $\mathrm{~V}$ & IV & I & 1 & IHS \\
\hline 17 & I & II & I & 1 & NHS \\
\hline 22 & I & IV & I & 1 & NHS \\
\hline 25 & I & IV & I & 1 & NHS \\
\hline 29 & I & II & I & 4 & IHS \\
\hline 37 & I & IV & I & 2 & IHS \\
\hline 30 & III & II & I & 1 & NHS \\
\hline 28 & I & III & I & 2 & NHS \\
\hline 29 & I & II & II & 1 & IPE \\
\hline
\end{tabular}

At the time of first diagnosis of PPCM, in G II patients, 4 were in FC I and 1 in FC II. One of the patients had two subsequent pregnancies and all pregnancies were well tolerated. The eldest patient, who was at that time in FC II, deteriorated into FC IV and died of cardiac failure 8 years after the last pregnancy, 13 years after the first diagnosis of PPCM. The remaining five patients are still alive, 3 in FC I and 2 in FC II. One (16.7\%) of the patients who was at first in FC $\mathrm{I}$, after the subsequent pregnancy deteriorated into FC II, showing a worsening of clinical status.

In regard to the fetus status, no alterations were seen among the 16 pregnancies observed (all children were born on due time, without any malformations, with adequate weight, height and development). The following pregnancy occurred 3 to 7 months (mean 41 months or 3.7 years) after complete recovery of PPCM. All patients were administered anticoagulants after delivery, and no thromboembolic phenomena was observed.

\section{Discussion}

PPCM is a syndrome of unknown etiology, with a variety of hypothesis proposed to explain it, none yet widely accepted ${ }^{1-4}$. Due to its particular epidemiological features, it has been characterized as a distinct disease, occurring most frequently after delivery, generally one week to six months postpartum ${ }^{1-14}$. Clinical deterioration occurs mainly in the last three months of pregnancy, as opposed to other diseases, which tend to produce an earlier deterioration (first or second trimester) due to the increase in blood volume consequent to cardiocirculatory changes associated with pregnancy ${ }^{1-14}$.

It has been speculated that viral infections might be associated to PPCM. No evidence of a viral infection or antibody viral titers were detected, however. Other hypothesis are: 1) a decreased activity of suppressor T-cells by progesterone, predisposing a cardiotropic viral infection; 2) an abnormal maternal autoimmune reaction producing antibodies against the fetus, placenta or myometrium antigens, cross-reacting with maternal myocardium; 3 ) a hormonal effect leading to thickening of the coronary artery intima layer and subsequent small-vessel coronary artery disease; 4) a nutritional deficiency; 5) genetic determination (familial predisposition); 6) local habits (such as observed in African tribes) ${ }^{15-17}$.

The reported incidence of peripartum myocarditis varies from 29 to $100 \%$. Immunossupression has been recommended for patients who do not respond to conventional therapy in 6 weeks. Clinical course is also variable; African populations seem to show rapid improvement in clinical condition after therapy, whereas in other countries, a severe impairment of systolic function with cardiac chamber dilation and decrease in left ventricular ejection fraction has been reported ${ }^{5}$. Improvement in left ventricular function and heart size is about $50 \%$ in 6 to 12 months ${ }^{15}$, however, complete clinical recovery has been observed by Meadows ${ }^{14}$ in two thirds of the patients.

Demakis and coworkers ${ }^{1}$ have shown that patients who had heart size normalized within 6 months presented a $14 \%$ mortality, unrelated to myocardial disease (due to cervical carcinoma and renal disorders). Nevertheless, $85 \%$ of patients (11/13 patients) with persistent cardiomegaly died of congestive heart failure in a mean of 4.7 years, three of them after deteriorating ventricular function in a subsequent pregnancy and 8 due to repetitive episodes of decompensation of chronic heart failure. Walsh et al. confirmed these findings ${ }^{18}$, demonstrating that among 12 patients with persistent cardiomegaly, 9 (75\%) died of congestive heart failure in 29 months (range 4 - 58months). However, a lower mortality rate ( $45 \%$ ) has been described by Seftel and Susser ${ }^{19}$, who observed 5 deaths in a group of 11 patients with persistent increase in heart size. In the present study, only one patient with persistent cardiomegaly died (1/6 $16.7 \%$ ), and that occurred 5 years after the last pregnancy. This finding is consistent with the use of more effective therapy for heart failure, such as ACE inhibitors. The leading mortality causes in patients with persistent cardiomegaly are: thromboembolic events, mainly pulmonary thromboembolism ${ }^{15-16}$, refractory heart failure and ventricular arrhythmia ${ }^{16}$. 
Meadows ${ }^{14}$ has reported a group of 10 patients with PPCM and subsequent pregnancy in which 6 (60\%) were associated with relapses, 2 died of congestive heart failure, one remained with cardiomegaly and chronic heart failure, while three of them had improvement of clinical status. Additionally, similar results have been observed by Walshe et al. who showed that in a group of 6 patients with MMCP and subsequent pregnancy, 2 had severe deterioration of clinical status while 4 had some degree of clinical deterioration. Among 27 patients, 14 normalized heart size and eight had a subsequent pregnancy. Two of these had progressive worsening of cardiac failure, but eventually recovered cardiac function. One patient, however, had clinical deterioration after twenty years due to hypertensive cardiomyopathy. Among the 13 remaining patients with cardiomegaly, 6 had a subsequent pregnancy. Three of these worsened left ventricular dysfunction and died. No change was observed in cardiac status, though ${ }^{1}$.

Burch at al suggest that prolonged bed rest has an important role in the treatment of PPCM. They treated 18 patients with 26 subsequent pregnancies, 11 of whom had no bed rest compared to 15 who were restricted to bed from the last 4 months of pregnancy up to 2 months post partum. They noticed that, in the group with strict bed rest, no patient presented with PPCM, with relapses occurring in $88 \%$ of patients in the group without bed rest (9/11). Although none of the patients with bed rest developed cardiomegaly, 3 patients in each group had fetal loss ${ }^{20}$.

In spite of the satisfactory results produced by bed rest and the reports of complete recovery after an additional episode of PPCM, the potential risk of worsening of cardiac function and death are still very high for subsequent pregnancies, thus confirming the recommendation to avoid subsequent pregnancies after a first episode of $\mathrm{PPCM}^{17}$.

Our approach was to discourage a subsequent pregnancy, according to Benchimol et $\mathrm{al}^{3}$, as well as Julian and Szekely ${ }^{17}$, while at the same time we were tempted to adopt the approach of Demakis et al ${ }^{1}$, who suggested that patients who had heart size restored to normal could have a subsequent pregnancy.

It is not clear yet if relapses in subsequent pregnancies are due to clinical deterioration of cardiac failure or if they are a reactivation of the disease. Relapses have also been reported to occur in normal pregnancies, suggesting a reactivation of an underlying process ${ }^{21}$.

The incidence of PPCM is higher in women with twin pregnancies, with eclampsia, multiparous, over 30 years old and black. These are thus the most commonly reported risk factors for this cardiomyopathy ${ }^{15}$.

Our group of patients consisted of a young population, with a mean age 26 years (ranging from 16 to 41 ) at the time of first diagnosis of PPCM, and 32 years at the time of subsequent pregnancy. Seven patients were over 30 years during the subsequent pregnancy, nine were nonwhite $(68.1 \%)$, one had preeclampsia and none had a twin gestation. Accordingly, the major risk factors for PPCM relapse were race (9/12 non-white) and age (7/12 over 30 years). Despite the fact that most patients had low social economical level, no nutritional deficiency contributing to myocardial dysfunction, as proposed by Walsh et al. ${ }^{18}$, was observed. That finding was not observed by Seftel and Susser, either ${ }^{19}$.

Tubal ligation was offered as a means of contraception for patients with left ventricular dysfunction after one year of the first diagnosis of PPCM ${ }^{3,11,17}$. However until they decided for it, they were advised to avoid sexual intercourse during fertile periods, and partners were told to use condoms; eventually, intrauterine devices were recommended. However, recommendations were forgotten as soon a clinical conditions improved, in spite of being aware of the risks of a subsequent pregnancy. These patients often use medication contraindicated for the fetus (such as ACE inhibitors and oral anticoagulants), they missed consultations after realizing they were pregnant, and sought emergency medical assistance when fearing for fetal risks. After becoming conscious of the risks of the new pregnancy, they became very serious about the consultations. We have followed 16 pregnancies in 12 patients, two patients with 2 subsequent pregnancies and one with 3 . Since the first pregnancy was devoid of risk and no deterioration of left ventricular function was observed, it was decided to inform the couple about possible risks of a pregnancy, explaining there was no general agreement about that up to then.

Preeclampsia, a complication observed after the $20^{\text {th }}$ week of pregnancy, was observed in one of the patients (16.6\%), who belonged to the group with normalized heart size and complete recovery of PPCM. However, the patient was black, 30 years old and had a positive family history for hypertension. She presented with progressive deterioration of cardiac function, progressing from FC I to II, persisting in FC II after the new pregnancy. Echocardiography showed the following findings at the onset of PPCM: LVSD - $5.5 \mathrm{~cm}$; LVDD - $6.4 \mathrm{~cm}$; left atrium: $3.2 \mathrm{~cm}$. During the pregnancy when she had preeclampsia ventricular diameters remained the same, but atrial size increased to $5.6 \mathrm{~cm}$.

According to Benchimol et al ${ }^{3}$ and Julian and Szekely ${ }^{17}$, arterial blood pressures in PPCM can be high, normal or low. The association with pregnancy-induced hypertension $(\mathrm{PIH})$ is common. Eclampsia is also a disease of unknown etiology ${ }^{22}$, however, the pathologic changes found in maternal organs are due to generalized vasospasm, with hemodynamic, renal (sodium retention and proteinuria) and hematological manifestations. The incidence is increased in young primigravidas (though it also affects women over 30 years), and multiple pregnancies, such as in $\mathrm{PPCM}^{22}$. One question still remains unanswered. Could PIH lead to PPCM, after a diffuse arteriolar vasospasm?

The difference between PIH and PPCM is that in PIH, after the termination of pregnancy and blood pressure control, there is no ventricular dysfunction left. Furthermore, right ventricular dysfunction is not observed in preeclampsia, as seen in PPCM ${ }^{22}$.

Spontaneous abortions have also been reported ${ }^{18,19}$, but that finding has not been observed in the present study. 
We have adopted an approach modified by Burch et al., recommending bed rest in the last two months of a subsequent pregnancy and the first 2 months of puerperium. We do not recommend absolute bed rest, but limited household physical activities, such as washing and ironing. Resting after the meals is part of the recommendations.

Among the patients with persistent cardiomegaly (4 in FC I and 2 in FC II), one had two subsequent pregnancies and in one, cardiac function deteriorated, becoming FC II after pregnancy. No evidence of infection was detected in this patient. Among the patients who were in FC II, only the eldest had a worse in cardiac function, and that happened 5 years after pregnancy, that is, 13 years after PPCM. She became FC III and eventually FC IV, dying of refractory heart failure due to severe left ventricular dysfunction. Left ventricular chambers measured at that time $7.2 \mathrm{~cm}$ (LVEDD), $6.2 \mathrm{~cm}$ (LVESD) and $4.1 \mathrm{~cm}(\mathrm{LA})$.

The interval between pregnancies ranged from 3 to 7 years (mean 3.7 years, 41 months). After the diagnosis of PPCM, most patients were in FC IV, but they rigorously followed medical recommendations, not only regarding drug therapy, but also the advice to avoid physical activities and a new pregnancy. Right after they became FC I, they disregarded medical recommendations. As soon as they became asymptomatic, they forgot to follow various recommendations, among them to avoid pregnancy. Thus, it could be observed that, after the third year, the number of pregnancies increased. Oakley suggests that patients with complete recovery and normal heart size should wait 3 to 5 years before a new pregnancy. Benchimol et al. ${ }^{3}$ have reported some changes to last for 6 months after the onset of the disease, not infrequently lasting longer, even when there are clinical signs of recovery. Since myocardial changes might still occur in the first two years, a three year interval would be safer to prevent new cardiovascular and immunological maternal abnormalities.

Subsequent pregnancies are well tolerated in patients with complete or partial cardiac functional recovery who are in FC I and II. Complications such as preeclampsia and death could not be correlated with PPCM. These findings allow us to suggest a more flexible approach for patients with PPCM: patients who have had their heart size normalized are not advised against conception any longer after a three-year interval from the onset of the disease, whereas those with persistent cardiomegaly are told about the risks of a future pregnancy and tubal ligation is recommended, with the final decision left for discussion among the patient, as well as their family and physicians. Clinical follow-up is provided, however, for those who become pregnant earlier than medical recommendation. Termination of gestation is not indicated.

\section{References}

1. Demakis JG, Rahimtoola SH, Sutton GC, et al. Natural course of peripartum cardiomyopathy . Circulation 1971; 44: 1053-61.

2. O'Connell JB, Costanzo-Nordin MR, Subrananian R, et al. Peripartum cardiomyopathy: clinical, hemodynamic, histologic and prognostic characteristics. J Am Coll Cardiol 1986; 8: 52-6.

3. Benchimol AB, Benchimol CB, Albanesi F. ${ }^{\circ}$ FM. Cardiomiopatia periparto. Arq Bras Cardiol 1988; 51: 107-15.

4. Midei MG, DeMent SH, Feldman NA. Peripartum myocarditis and cardiomyopathy. Circulation 1990; 81: 922-8.

5. Resende MCV, Barretto ACP, Medeiros C, et al. A miocardiopatia periparto é uma doença benigna? Análise de possíveis fatores prognósticos. Rev Soc Cardiol ESP 1992; 2: 76-80.

6. Hsieh CC, Chiang CW, Hsieh TT. Peripartum cardiomyopathy. JPN Heart J 1992; 33: 343-49.

7. Ianni BM, Barretto ACP. Miocardiopatias não inflamatórias do adulto. In: Silva MAD Doença do Miocárdio. São Paulo: Sarvier, 1995: 147-61.

8. Cunningham FG, Pritchard JA, Hankins GDV. Peripartum heart failure: idiopathic cardiomyopathy or compounding cardiovascular events? Obstet Gynecol 1986; 67: 157-67.

9. Oakley CDG. Pregnancy and heart disease. In: Julian D, et al. (ed.) Diseases of Heart , $2^{\mathrm{a}}$ ed. London: WB Saunders Co, 1996: 1336.

10. Elkayam U. Pregnancy and cardiovascular disease. In: Braunwald E (ed.) - Heart Disease: A textbook of cardiovascular medicine, $5^{\mathrm{a}}$ ed. Philadelphia: WB Saunders Co, 1997: 1851-52.
11. St. John Sutton MSJ, Cole P, Plappert M, et al. Effects of subsequent pregnancy on left ventricular function in peripartum cardiomyopathy. Am Heart J 1991; 121 : 1776-8.

12. Ravokishore AG, Kaul UA, Sethi KK, Khalilullah M. Peripartum cardiomyopathy: Prognostic variables at initial evaluation. Int J Cardiol 1991; 32: 377-82.

13. Nwosu EC, Burke MF. Cardiomyopathy of pregnancy. Br J Obstet Gynecol 1992; 100: 1145-50.

14. Meadows WR. Idiopathic myocardial failure in the last trimester of pregnancy and puerperium. Circulation 1957; 15: 903-14

15. Homans DC. Peripartum cardiomyopathy. N Engl J Med 1985; 312: 1432-7.

16. Mc Mullan MR, Moore CK, O'Connell JB. Diagnosis and management of peripartum cardiomyopathy. Hospital Practice 1993; 89-104.

17. Julian DG, Szekely P. Peripartum cardiomyopathy. Prog Cardiovas Dis 1985; 27: 223-40.

18. Walsh JJ, Burch GE, Black WC, Ferrans VJ, Hibbs RG. Idiopathic myocardiopathy of the puerperium (postpartal heart disease). Circulation 1965; 32: 19-31.

19. Seftel H, Susser M. Maternity and myocardial failure in African women. Br Heart J 1961; 23: 43-52.

20. Burch GE, McDonald CD, Walsh JJ. The effect of prolonged bed rest on postpartal cardiomyopathy. Am Heart J 1971; 81: 186-201.

21. Wilmer G. Pospartal heart disease. South Med J 1963; 56: 803-11.

22. Kahhale S, Zugaib M. Síndromes Hipertensivas na Gravidez, $1^{\text {a }}$ ed., Rio de Janeiro: Editora Atheneu, 1995. 